\title{
EFEITO DA TEMPERATURA NA PARTIÇÃO DE ALFA- LACTALBUMINA EM SISTEMAS AQUOSOS BASEADOS EM LÍQUIDOS IÔNICOS
}

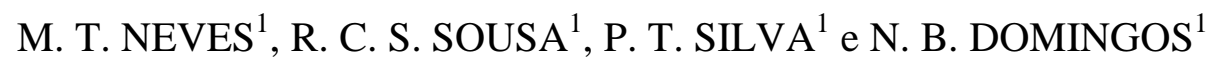 \\ ${ }^{1}$ Universidade Federal de Viçosa, Departamento de Química e Engenharia Química \\ E-mail para contato: rita.sousa@ufv.br
}

\begin{abstract}
RESUMO - Os sistemas aquosos bifásicos (SAB) baseados em líquidos iônicos (LI) têm sido aplicados em processos de purificação devido às suas "características verdes". O objetivo deste trabalho foi estudar a partição de alfalactalbumina do soro de leite em SAB formados por $\mathrm{LI}\left(\mathrm{C}_{4} \mathrm{minCl}\right)$ e fosfato de potássio em função da temperatura $\left(5,25,35\right.$ e $\left.45^{\circ} \mathrm{C}\right)$. A análise termodinâmica foi realizada pela aproximação de van't Hoff. As quantidades de LI e sal foram determinadas de acordo com dados de equilíbrio de fases disponíveis em literatura. Após a pesagem dos componentes, os tubos foram agitados, centrifugados e deixados em repouso na temperatura de trabalho. A quantificação da proteína nas fases foi realizada por espectrofotometria. O coeficiente de partição $(\mathrm{K})$ foi obtido pela relação entre as concentrações de proteína na fase superior (rica em LI) e inferior (rica em sal) e variou de 1,50 a 57,10. A partição da proteína para fase superior dos SAB foi espontânea e entalpicamente dirigida. Os resultados obtidos despertam o interesse por estudos posteriores de forma a elucidar o tipo de interação envolvida.
\end{abstract}

\section{INTRODUÇÃO}

O soro de leite é o co-produto mais abundante da indústria de laticínios e é rico em proteínas de alto valor nutricional, funcional e tecnológico. Entre essas proteínas, encontramos a alfalactalbumina, que também é encontrada no leite humano. Ela desempenha importante função na prevenção de úlcera gástrica (Matsumoto et al., 2001) e possui elevado teor de triptofano, sendo capaz de elevar o triptofano sanguíneo (Heine et al., 1991), entre outras vantagens e aplicações. A recuperação e valorização dessa proteína são, portanto, de grande interesse para ambos os campos acadêmico e industrial.

Os Sistemas Aquosos Bifásicos ( $\mathrm{SAB}$ ) têm adquirido importância e sucesso crescente no que diz respeito à concentração, isolamento e separação de proteínas. Estes sistemas são constituídos por duas fases líquidas imiscíveis, que promovem a separação de biomoléculas em condições amenas e em um ambiente adequado, de forma que sejam preservadas as suas principais características. A alta concentração de água em tais sistemas favorece a estabilidade das proteínas durante a separação, quando comparados com sistemas de extração líquida tradicionais, compostos com solventes orgânicos. Apresentam também vantagens como rapidez da separação, baixo custo e possibilidade de aplicação em grande escala (Ventura et al., 2011; 2009). 
SAB convencionais são formados por uma mistura de dois polímeros, ou um polímero e um sal inorgânico com água. No entanto, a maioria dos polímeros utilizados em SAB reportados em literatura apresenta alta viscosidade (Perumalsamy et al., 2007). Por esse motivo, as soluções formadas por esses polímeros geralmente são opacas, o que pode interferir nas análises qualitativas e quantitativas dos compostos extraídos nos sistemas.

Assim, nos últimos anos, SAB baseados em líquidos iônicos (LI) têm sido aplicados em processos de biopurificação e bioextração oferecendo diversas vantagens como a estabilidade de proteínas e enzimas além de serem substancialmente menos viscosos que os polímeros usados tipicamente (Ventura et al., 2011; 2009; Jiang et al., 2009; Dreyer, 2008; Claudio et al., 2010; Louros et al., 2010). Além disso, os SAB formados por LI e sais inorgânicos apresentam maiores taxas de recuperação de proteínas modelo do que os métodos empregando SAB formados por polímero (PEG) e sal (Pei et al., 2009).

Os LI são sais líquidos a temperatura ambiente e são normalmente formados por um cátion orgânico relativamente grande e um ânion orgânico ou inorgânico relativamente pequeno. Estas substâncias diferem dos sais comuns por apresentarem uma fraca interação interiônica, a qual resulta em uma baixa energia do retículo cristalino e baixa temperatura de fusão (Cassol, 2007). O estudo dos LI estáveis começou na década de 60, mas nos últimos dez anos tem havido um crescimento exponencial de publicações relacionadas a trabalhos aos mesmos.

Neste trabalho, foi estudada a extração da alfa-lactalbumina presente no soro de leite, em sistemas aquosos bifásicos constituídos pelo LI 1-butyl-3-methylimidazolium chloride $\left(\mathrm{C}_{4} \mathrm{minCl}\right)$, pelo sal fosfato de potássio dibásico anidro $\left(\mathrm{K}_{2} \mathrm{HPO}_{4}\right)$ e água, a temperaturas de $5,25,35$ e $45^{\circ} \mathrm{C}$, com quatro diferentes concentrações do LI (18, 20, 22 e 24\%).

\subsection{Proteínas do soro de leite}

A produção de soro de leite aumentou acentuadamente nas últimas décadas, juntamente com a produção de queijo (Dermiki et al., 2008), sendo estimada na ordem dos 160 milhões de toneladas por ano (Magalhães et al., 2010). O soro de leite, quando lançado em cursos d'água, apresenta potencial poluidor aproximadamente 100 vezes maior que o do esgoto doméstico (Porto et al., 2005). Cada vez mais, portanto, a legislação ambiental exige das indústrias de laticínios um plano de tratamento ou reaproveitamento deste soro (Lira et al., 2009).

Os produtos de soro apresentam a importante vantagem de possuírem propriedades funcionais, além de ser uma fonte concentrada de nutrientes lácteos, sobretudo proteínas de elevado valor nutricional (Thamer e Penna, 2006). Cerca de $80 \%$ das proteínas do soro são constituídos por $\alpha$-lactalbumina ( $\alpha$-la), $\beta$-lactoglobulina $(\beta-\lg )$ e glicomacropeptídeo (GMP) (Graselli et al., 1997). Estas proteínas oferecem diversas aplicações industriais e apresentam as seguintes propriedades: atividade anti-carcinogênica; aumento da resistência do sistema imunológico e da longevidade do organismo; anti-hipertensivas, hipocolesterolêmicas; apresentam solubilidade em solventes alimentícios; capacidade de hidratação e de retenção de água; capacidade emulsificante; de formação de espumas/retenção de ar; de gelificação e de estabilizar emulsões e podem ser usadas como suplemento alimentício (Graselli et al., 1997; Sgarbieri, 1996). 


\subsection{Sistemas aquosos bifásicos baseados em líquidos iônicos}

Em tempos de melhoria da engenharia molecular, o desenvolvimento de novos e biocompatíveis métodos de extração para a separação e purificação de enzimas e proteínas vem ganhando importância crescente. Um método eficaz e economicamente viável para a separação e purificação de biomoléculas é a sua partição através de Sistemas Aquosos Bifásicos (SAB).

Os $\mathrm{SAB}$ são formados por espécies químicas que, quando misturadas em determinadas faixas de composição e temperatura, dividem-se em duas fases com composições diferentes, em equilíbrio termodinâmico. A purificação é resultado de uma partição diferenciada da moléculaalvo e impurezas entre as duas fases líquidas (Pessoa Jr. e Kilikian, 2005). Solutos se distribuem entre as duas fases, dependendo da sua afinidade relativa por cada uma das fases individuais. Esta distribuição é governada por um grande número de fatores, por exemplo: natureza e tamanho da partícula alvo; constituição, tamanho e estrutura molecular do polímero; temperatura; natureza do eletrólito e pH do sistema bifásico (Albertsson, 1986). Por isso, a predição e a interpretação da partição de macromoléculas em SAB demanda muitos estudos.

As vantagens desta técnica incluem seletividade favorável, baixo custo e adaptabilidade para o processamento contínuo da amostra e retenção da atividade biológica. No entanto, a maior parte dos polímeros utilizados na formação dos $\mathrm{SAB}$ possuem alta viscosidade e, normalmente, formam soluções aquosas opacas, que poderiam interferir na análise quantitativa e qualitativa dos compostos extraídos (Perumalsamy, 2007; Ventura et al., 2011).

Durante os últimos anos, os Líquidos Iônicos (LI) têm sido aplicados por processos de biopurificação, e possuem vantagens, tais como a melhoria da estabilidade da enzima, o substrato e/ou seletividade do produto, e supressão de reações secundárias foram observadas. Além disso, os SAB baseados em LI são substancialmente menos viscosos do que os típicos SABs à base de polímeros (Claudio; Louros, 2010; Ventura et al., 2011).

Estes compostos possuem propriedades físico-químicas muito interessantes, destacando-se as seguintes características: são usualmente líquidos em uma ampla faixa de temperatura próxima à temperatura ambiente; apresentam viscosidade relativamente baixa; não são inflamáveis; apresentam estabilidades térmica e eletroquímica maiores que solventes orgânicos clássicos; dissolvem inúmeros compostos orgânicos e inorgânicos; podem ser modulados pela seleção do cátion ou ânion; são estáveis ao ar; são facilmente recicláveis; são sintetizados a partir de reagentes comerciais acessíveis e utilizando métodos sintéticos de relativa simplicidade (Cassol, 2007).

Desta maneira, estas substâncias oferecem grande potencial de aplicação em bioprocessos para a separação das macromoléculas de interesse de matrizes biológicas complexas (Du et al., 2007).

\section{MATERIAL E MÉTODOS}

O líquido iônico utilizado nestes experimentos foi o 1-butyl-3-methylimidazolium chloride $\left(\mathrm{C}_{4}\right.$ mimCl$)$ e o sal inorgânico, fosfato de potássio dibásico anidro $\left(\mathrm{K}_{2} \mathrm{HPO}_{4}\right)$. A alfa-lactalbumina foi adquirida da DAVISCO Food Ingredientes International (Eden Praire, EUA). Além disso, água deionizada foi utilizada para formação dos sistemas. 
Os SAB foram preparados de acordo com dados de equilíbrio encontrados em literatura (Ventura et al., 2011), de modo a se obter sistemas com quatro diferentes concentrações em massa de LI $(18 \%, 20 \%, 22 \%$ e $24 \%)$.

A Tabela 1 mostra a quantidade (\% em massa) adicionada de cada componente do sistema, para formar um SAB de massa total 12 gramas. As concentrações em massa de LI utilizadas foram escolhidas de modo que houvesse formação de fases, e que o volume da fase superior fosse o mais próximo possível da fase inferior, de modo que a diferença de volume das fases pudesse ser desconsiderada como fator determinante do coeficiente de partição.

Tabela 1 - Composição mássica dos SAB para diferentes valores de concentração do LI

\begin{tabular}{|c|c|c|c|c|}
\hline \multicolumn{5}{|c|}{ Concentração de LI (\% m/m) } \\
\hline & $18 \%$ & $20 \%$ & $22 \%$ & $24 \%$ \\
\hline $\mathrm{m}_{\text {sal }}(\mathrm{g})$ & 3,2307 & 2,9819 & 2,7330 & 2,4842 \\
\hline $\mathrm{m}_{\text {água }}(\mathrm{g})$ & 6,6093 & 6,6181 & 6,6270 & 6,6358 \\
\hline $\mathrm{m}_{\text {LI }}(\mathrm{g})$ & 2,1600 & 2,4000 & 2,6400 & 2,8800 \\
\hline
\end{tabular}

Os sistemas foram preparados em tubos de centrífuga graduados e calibrados, e em cada sistema eram adicionados sequencialmente, utilizando-se uma balança analítica, $10 \mathrm{mg}$ da proteína, o fosfato de potássio, a água e o líquido iônico. Cada sistema foi preparado em duplicata.

Após a preparação, os $\mathrm{SAB}$ foram deixados em repouso por aproximadamente 12 horas, à temperatura desejada $\left(5,25,35\right.$ ou $\left.45^{\circ} \mathrm{C}\right)$, para que o equilíbrio fosse atingido. Em seguida, o volume de cada fase foi medido e elas foram separadas, com o auxílio de uma seringa. A determinação da concentração de proteína nas fases foi feita em espectrofotômetro (Cary 50 Probe, Varian, EUA), a $280 \mathrm{~nm}$. Uma curva padrão da proteína nas fases foi previamente preparada, e assim a concentração pôde ser determinada. O coeficiente de partição (Equação 1) para cada uma das concentrações de LI, em cada temperatura em estudo foi calculado.

$$
K=\frac{C \text { sup }}{\operatorname{Cinf}}
$$

$\mathrm{Na}$ equação $1, \mathrm{C}_{\text {sup }}$ e $\mathrm{C}_{\text {inf }}$ representam, respectivamente, a concentração da proteína nas fases superior (rica em LI) e na fase inferior (rica em sal inorgânico).

Do ponto de vista termodinâmico, a extração da proteína pode ser vista como um processo de transferência da proteína da fase rica em sal (inferior) para a fase rica em LI (fase superior). A Energia de Gibbs de um processo de transferência a uma dada temperatura $(\mathrm{T})$ pode ser calculada a partir da equação 2, utilizando o coeficiente de partição $(\mathrm{K})$. 


$$
\Delta G_{T}=-R T \ln K
$$

em que, $\mathrm{K}$ é o coeficiente de partição da proteína entre as fases, T é a temperatura (em Kelvin) e $\mathrm{R}$ é a constante dos gases. A variação de entalpia e entropia associadas à partição da proteína podem ser calculadas, respectivamente, a partir da inclinação e do intercepto da equação linear entre ln K e 1/T (Equação 3).

$$
\ln K=\frac{-\Delta H_{T}}{R T}+\frac{\Delta S_{T}}{R}
$$

\section{RESULTADOS E DISCUSSÃO}

A concentração das proteínas nas fases foi determinada por meio de leituras de absorbância em espectrofotômetro a $280 \mathrm{~nm}$. Uma curva padrão de concentração de proteína versus absorbância foi previamente construída. Verificou-se a dificuldade em realizar a leitura da absorbância para a fase inferior (rica em sal), dado os valores obtidos, de modo geral, eram negativos e baixos, sugerindo um erro do próprio equipamento. Para a fase superior, a leitura da absorbância estava de acordo com o esperado. A concentração de proteína da fase inferior foi determinada por meio de um balanço de massa: conhecidos o volume e a concentração de proteína da fase superior, e sabendo-se que em cada SAB foram adicionados $10 \mathrm{mg}$ de proteína, foi possível determinar a massa de proteína restante na fase inferior. Com a massa de proteína e o volume da fase, calculou-se a concentração nesta fase.

As Tabelas 2 a 6 mostram os resultados de partição da alfa-lactalbumina nos SAB baseados no LI $\mathrm{C}_{4} \mathrm{minCl}$ e no sal $\mathrm{K}_{2} \mathrm{HPO}_{4}$ para cada temperatura estudada.

Tabela 1 - Partição de alfa-lactalbumina em SAB a $5^{\circ} \mathrm{C}$

\begin{tabular}{|c|c|c|c|}
\hline \multirow{2}{*}{ Concentração LI } & \multicolumn{2}{|c|}{ Concentração de alfa $(\mathrm{mg} / \mathrm{mL})$} & \multirow{2}{*}{ Coeficiente de partição $(\mathrm{K})$} \\
\cline { 2 - 3 } & Fase Superior & Fase Inferior & 17,4443 \\
\hline $18 \%$ & 2,0842 & 0,1195 & 15,8188 \\
\hline $20 \%$ & 1,9012 & 0,1202 & 13,5804 \\
\hline $22 \%$ & 1,7014 & 0,1253 & 11,5051 \\
\hline $24 \%$ & 1,5754 & 0,1369 & \\
\hline
\end{tabular}

Tabela 3 - Partição de alfa-lactalbumina em SAB a $15^{\circ} \mathrm{C}$

\begin{tabular}{|c|c|c|c|}
\hline \multirow{2}{*}{ Concentração LI } & \multicolumn{2}{|c|}{ Concentração de alfa $(\mathrm{mg} / \mathrm{mL})$} & \multirow{2}{*}{ Coeficiente de partição (K) } \\
\cline { 2 - 3 } & Fase Superior & Fase Inferior & \\
\hline $18 \%$ & 2,1023 & 0,1037 & 20,2659 \\
\hline $20 \%$ & 2,0157 & 0,1046 & 19,2705 \\
\hline $22 \%$ & 1,7182 & 0,1042 & 16,4820 \\
\hline $24 \%$ & 1,5572 & 0,1642 & 9,4832 \\
\hline
\end{tabular}


Tabela 4 - Partição de alfa-lactalbumina em SAB a $25^{\circ} \mathrm{C}$

\begin{tabular}{|c|c|c|c|}
\hline \multirow{2}{*}{ Concentração LI } & \multicolumn{2}{|c|}{ Concentração de alfa $(\mathrm{mg} / \mathrm{mL})$} & \multirow{2}{*}{ Coeficiente de partição (K) } \\
\cline { 2 - 3 } & Fase Superior & Fase Inferior & \\
\hline $18 \%$ & 1,9691 & 0,2191 & 8,9882 \\
\hline $20 \%$ & 1,7802 & 0,2424 & 7,3436 \\
\hline $22 \%$ & 1,6094 & 0,2399 & 6,7076 \\
\hline $24 \%$ & 1,5034 & 0,2449 & 6,1396 \\
\hline
\end{tabular}

Tabela 5 - Partição de alfa-lactalbumina em SAB a $35^{\circ} \mathrm{C}$

\begin{tabular}{|c|c|c|c|}
\hline \multirow{2}{*}{ Concentração LI } & \multicolumn{2}{|c|}{ Concentração de alfa $(\mathrm{mg} / \mathrm{mL})$} & \multirow{2}{*}{ Coeficiente de partição (K) } \\
\cline { 2 - 3 } & Fase Superior & Fase Inferior & \\
\hline $18 \%$ & 1,6028 & 0,5360 & 2,9902 \\
\hline $20 \%$ & 1,4822 & 0,5435 & 2,7272 \\
\hline $22 \%$ & 1,3914 & 0,5119 & 2,7180 \\
\hline $24 \%$ & 1,1982 & 0,7026 & 1,7054 \\
\hline
\end{tabular}

Tabela 6 - Partição de alfa-lactalbumina em SAB a $45^{\circ} \mathrm{C}$

\begin{tabular}{|c|c|c|c|}
\hline \multirow{2}{*}{ Concentração LI } & \multicolumn{2}{|c|}{ Concentração de alfa $(\mathrm{mg} / \mathrm{mL})$} & \multirow{2}{*}{ Coeficiente de partição (K) } \\
\cline { 2 - 3 } & Fase Superior & Fase Inferior & \\
\hline $18 \%$ & 2,1149 & 0,0928 & 22,7796 \\
\hline $20 \%$ & 1,9858 & 0,0348 & 57,1043 \\
\hline $22 \%$ & 1,6505 & 0,1887 & 8,7484 \\
\hline $24 \%$ & 1,5110 & 0,2334 & 6,4727 \\
\hline
\end{tabular}

Os resultados apresentados nas Tabelas 2 a 6 mostram que a extração da alfa-lactalbumina de soro de leite nestes SAB se mostrou eficiente para todas as temperaturas estudadas. Na maioria dos casos, o coeficiente de partição diminui com o aumento da concentração do LI.

De acordo com estudos prévios, observa-se que a dependência dos coeficientes de partição em relação a temperatura depende largamente do soluto e do sistema ternário estudado. Pei et al. (2009) reportaram que a temperatura influencia fortemente a eficiência de extração de proteínas.

Usando as Equações 2 e 3 supracitadas, obtemos os valores de variação de energia livre de Gibbs, de entalpia e de entropia resultante da transferência da alfa-lactalbumina no SAB. Esses valores podem ser vistos na Tabela 7 . 
Tabela 7 - Propriedades termodinâmicas da transferência de alfa-lactalbumina no SAB

\begin{tabular}{|c|c|c|c|c|}
\hline $\mathrm{T}(\mathrm{K})$ & $\mathrm{K}^{*}$ & $\Delta \mathrm{G}_{\mathrm{T}}(\mathrm{kJ} / \mathrm{mol})$ & $\mathrm{T} \Delta \mathrm{S}_{\mathrm{T}}(\mathrm{kJ} / \mathrm{mol})$ & $\Delta \mathrm{H}_{\mathrm{T}}(\mathrm{kJ} / \mathrm{mol})$ \\
\hline 278 & 14,59 & $-6,19$ & $-35,59$ & $-42,5$ \\
\hline 288 & 16,38 & $-6,69$ & $-36,87$ & \\
\hline 298 & 7,29 & $-4,92$ & $-38,15$ & \\
\hline 308 & 2,54 & $-2,38$ & $-39,43$ & \\
\hline 318 & 13,78 & $-6,93$ & $-40,71$ & \\
\hline
\end{tabular}

* Os valores apresentados são a média dos valores de $\mathrm{K}$ para as diferentes concentrações de LI.

Os valores de $\Delta \mathrm{G}_{\mathrm{T}}, \Delta \mathrm{H}_{\mathrm{T}}$ e $\Delta \mathrm{S}_{\mathrm{T}}$ são apresentados na Tabela 7 . Os valores de $\Delta \mathrm{G}_{\mathrm{T}}$ são negativos para todos os $\mathrm{SAB}$ indicando que o processo de transferência da alfa-lactalbumina para fase superior, rica em LI, é espontâneo. Além disso, os valores de $\Delta \mathrm{H}_{\mathrm{T}}$ negativos indicam que é um processo exotérmico o que reflete em interações favoráveis do tipo proteína-LI. $\mathrm{O}$ valor de $\Delta \mathrm{H}_{\mathrm{T}}$, maior que $\mathrm{T} \Delta \mathrm{S}_{\mathrm{T}}$, indica que o processo é entalpicamente dirigido (Claudio et al., 2010).

\section{CONCLUSÃO}

Neste trabalho foi demonstrada a partição de alfa-lactalbumina do soro de leite em sistema aquoso bifásico constituído por um líquido iônico e uma fase aquosa salina. Os resultados obtidos indicam o uso de líquido iônico $\mathrm{C}_{4} \mathrm{mimCl}$ como um solvente alternativo para substituir os solventes orgânicos tradicionais em extração líquido-líquido para recuperação de proteínas do soro de leite. O processo de transferência da proteína para fase superior dos SAB é espontâneo e entalpicamente dirigido. Este novo sistema de extração é um método promissor para ser empregado na separação e pré-concentração das proteínas de soro de leite.

\section{REFERÊNCIAS}

CASSOL, C. C. Líquidos iônicos em processos de extração seletiva de compostos aromáticos, nitrogenados e sulfurados em frações do petróleo. Dissertação de Mestrado, Universidade Federal do Rio Grande do Sul, Instituto de Química, 2007.

ClÁUdiO, A. F. M.; FREIRE, M. G.; FREIRE, C. S. R.; SILVESTRE, A. J. D.; COUTINHO, J. A. P., Extraction of vanillin using ionic-liquid-based aqueous two-phase systems, Sep. Purif. Technol. v.75, p. 39-47, 2010.

DERMIKI, M.; NTZIMANI, A.; BADEKA, A.; SAVVAIDIS, I.N.; KONTOMINAS, M.G. Shelflife extension and quality attributes of the whey cheese "Myzithra Kalathaki" using modified atmosphere packaging. Food Sci. Technol., v. 41, p. 284-294, 2008.

DREYER, S.; KRAGL, U. Ionic liquids for aqueous two-phase extraction and stabilization of enzymes, Biotechnol. Bioeng. v.99, p. 1416-1424, 2008.

GRASSELli, M., NAVARRO, A., FERNANDEZ, H. L., MIRANDA, M. V., CAMPERI, I., OSVALDO CASCONE. Que hacer com el suero de queso? Ciencia Hoy. v. 43, p. 27-35, 1997. 
HEINE, W. E, KLEIN, P. D, REEDS, P. J. The importance of $\alpha$-lactalbumin in infantile nutrition. J Nutr., v.121, p. 277-83, 1991.

JIANG, Y.; XI, H.; YU, J.; GUO, C.; LIU, H. Hydrophobic ionic liquids-assisted polymer recovery during penicillin extraction in aqueous two-phase system. Chem. Eng. J., v. 147, p. 22-26, 2009.

LIRA, H.L.; SILVA, M.C.D.; VASCONCELOS, M.R.S.; LIRA, H.L.; LOPEZ, A.M.Q. Microfiltração do soro de leite de búfala utilizando membranas cerâmicas como alternativa ao processo de pasteurização. Ciência e Tecnologia de Alimentos, v. 29, n.1, p. 33-37, 2009.

LOUROS , C. L. S. CLAUDIO, A. F. M. NEVES, C. M. S. S. FREIRE, M. G. MARRUCHO, I. M. PAULY J., COUTINHO , J. A. P. Extraction of biomolecules using phosphonium-based ionic liquids $+\mathrm{K}_{3} \mathrm{PO}_{4}$ aqueous biphasic systems, Int. J. Mol. Sci. v.11, p. 1777-1791, 2010.

MAGAlHÃES, K. T.; PEREIRA, M. A.; NICOLAU, A.; DRAGONE, G.; DOMINGUES, L.; TEIXEIRA, J. A.; SILVA, J. B. A.; SCHWAN, R. F. Production of fermented cheese wheybased beverage using kefir grains as starter culture. Evaluation of morphological and microbial vantages. Bioressource Technol., v. 101, p. 8843-8850, 2010.

MATSUMOTO H, SHIMOKAWA Y, USHIDA Y, TOIDA T, HAYASAWA H. New biological function of bovine alpha-lactalbumin: protective effect against ethanol and stress-induced gastric mucosal injury in rats. Biosci. Biotechnol. Bioch., v. 65, p. 1104-1111, 2001.

PEI, Y. JIANJI, W. WU, K. XUAN, X. LU, X. Ionic liquid-based aqueous two-phase extraction of selected proteins Separation and Purification Technology. Sep. Purif. Technol. v.64, p.288295, 2009.

PERUMALSAMY M., BATHMALAKSHMI A., MURUGESAN T. Experiment and correlation of liquid-liquid equilibria of an aqueous salt polymer system containing PEG6000 + sodium citrate. J. Chem. Eng. Data, v.52, p.1186, 2007.

PORTO, L. M.; SANTOS, R. C.; MIRANDA, T. L. S. Determinação das melhores condições operacionais do processo de produção da ricota. Boletim CEPPA, v. 23, n. 1, p. 173-182, 2005.

SGARBIERI, V.C. Propriedades fisiológicas-funcionais das proteínas do soro de leite, Revista de Nutrição, 17 (4), 1-8, 2004.

THAMER, K.G.; PENNA, A.L.B. Caracterização de bebidas lácteas funcionais fermentadas por probioticos acrescidas de prebioticos. Ciência e Tecnologia de Alimentos, v. 26, n. 3, p. 589595, 2006.

VENTURA, S.P.M.; NEVES, C.; FREIRE, M.G.; MARRUCHO, I.M.; OLIVEIRA, J.; COUTINHO, J.A.P. Evaluation of Anion Influence on the Formation and Extraction Capacity of Ionic-Liquid-Based Aqueous Biphasic Systems. J. Physical Chem. B, v. 113(27), p. 9304-9310, 2009.

VENTURA, S. P. M.; SOUSA, S. G.; SERAFIM, L.S.; LIMA, A. S.; FREIRE, M. G.; COUTINHO, J. A. P. Ionic Liquid Based Aqueous Biphasic System with Controlled pH: The Ionic Liquid Cation Effect. J. Chem. Eng. v.56, p. 4253-4260, 2011. 